\title{
Cerebral Venous Sinus Thrombosis as a Complication of a Microendoscopic Discectomy
}

\author{
R. Sommerstein ${ }^{a} \quad$ H.H. Jung ${ }^{b} \quad$ C. Knoblauch ${ }^{a}$ \\ ${ }^{a}$ Department of Internal Medicine, Cantonal Hospital Nidwalden, Stans, and ${ }^{b}$ Department of Neurology, \\ University Hospital Zurich, Zurich, Switzerland
}

Dear Sir,

An 18-year-old woman was referred to the local hospital by her primary care physician because of severe nausea accompanied by bifrontal headaches and photophobia.

Three days prior to admission, she had undergone microendoscopic discectomy (MED) at the level $\mathrm{L}_{5} / \mathrm{S}_{1}$ for subligamentous left paramedian disc hernia. The procedure was performed under spinal anesthesia with no intraoperative complications.

Postoperative mobilization was normal, and the patient was discharged in good health on the 2 nd postoperative day.

Increasing headache developed shortly after returning home, and the patient was then referred to this hospital. On admission, she complained of severe bifrontal headache that was worse when she was upright, nausea and photophobia. The patient was overweight (BMI of 28.3). There were no signs of optical disc swelling, elevated blood pressure or focal neurologic deficit besides dysesthesia on the lateral edge of the left foot interpreted as residual sign following disc herniation. Routine laboratory analyses were normal apart from a slightly elevated C-reactive protein at $10 \mathrm{mg} / \mathrm{l}$ (normal value $<5 \mathrm{mg} / \mathrm{l}$ ). Intra- cranial hypotension due to a CSF leak was suspected, and bed rest, prophylactic lowmolecular-weight heparin, intravenous rehydration and symptomatic antiemetic and analgesic therapy with metoclopramide and paracetamol were prescribed.

On the 2nd hospital day, postural headaches worsened with frequent vomiting.

Further laboratory tests on the 3rd hospital day remained unremarkable, with Creactive protein returning to normal. Neurological focus or signs of meningitis did not develop but the headaches became non-postural. As intracranial hypotension syndrome became unlikely, a CT scan of the brain was performed on the 6th hospital day. A 6-cm-long filling defect was detected in the left superior sagittal sinus, corresponding to a cerebral venous sinus thrombosis (CVST; fig. 1a). A subsequent contrast-enhanced MRI demonstrated unspecific and discrete meningeal enhancement on the $\mathrm{T}_{1}$-weighted scans. CVST was confirmed, and there was no evidence for a venous infarction (fig. 1b).

Upon detailed inquiry, a history of an earlier anterior spinal artery thrombosis was mentioned in the patient's younger brother. A workup for thrombophilia identified the patient as heterozygous for factor V Leiden. The patient was taking oral contraception. Therapeutic low-molecular-weight heparin and a 6-month course of warfarin were initiated. Six months later, repeated MRI scan showed complete clot resolution. The patient had no residual symptoms and warfarin therapy was discontinued.

In a recent review, the cause of CVST was described as typically plurifactorial [1]. This patient presented with multiple important risk factors for CVST: neurosurgery, oral contraception and a factor $\mathrm{V}$ Leiden mutation $[2,3]$. It is likely that a CSF leak following MED was the initial trigger for the clotting. The first symptoms and the generalized meningeal enhancement seen on the MRI scan most likely reflect signs of the intracranial hypotension syndrome [4]. This CSF leak may have led to a cascade of phenomena: firstly a downward shift of the intracranial contents due to lowered intracranial pressure, and then subsequent venous vasodilatation with endothelial alteration and stasis [5] initiating clot formation by classical Virchow triad. The subsequent nonpostural headaches and vomiting are best explained by increasing intracranial pressure in the context of the cerebral venous thrombosis.

\section{KARGER}

Fax +4161306 1234 E-Mail karger@karger.ch www.karger.com
Rami Sommerstein, MD

Department of Internal Medicine, Cantonal Hospital Zug

Landhausstrasse 11

CH-6340 Baar (Switzerland)

Tel. +41 41399 4228, Fax +41 41399 4021, E-Mail rami.sommerstein@ zgks.ch 

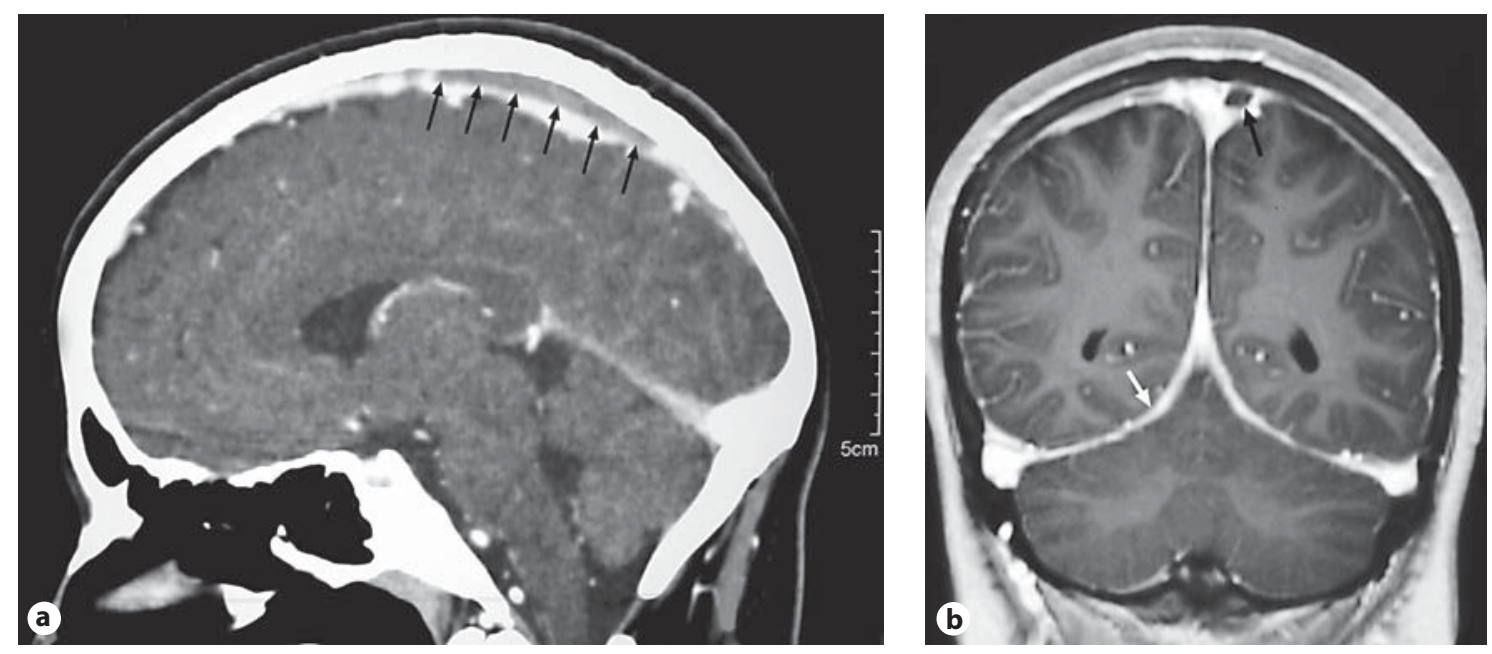

Fig. 1. a Contrast-enhanced sagittal 2-D CT reconstruction of the head: a filling defect $6 \mathrm{~cm}$ in length corresponding to a CVST is seen in the superior sagittal sinus (arrows). b Contrast-enhanced coronary $\mathrm{T}_{1}$-weighted MRI: discrete diffuse meningeal enhancement (white arrow) is seen. There is incomplete thrombosis in the left superior sagittal sinus, occluding one third of the lumen without evidence of adjacent venous infarction.

While the use of oral contraceptives (54.3\%) and thrombophilia (34.1\%) seems to be a frequent risk factor for CVST, mechanical precipitants are rare as an underlying condition being present in $4.5 \%$ of all the cases of CVST [6]. Within the causes of mechanical precipitants, neurosurgery was only present in $0.6 \%$ of all cases, and to our knowledge no cases were described after MED.

In conclusion, we described the complication of a CVST after MED of the lum- bar spine in a patient with multiple risk factors. Persisting headache in patients after lumbar manipulation should raise suspicion for CVST.

\section{Acknowledgements}

We would like to thank Tom Brinks for critical proofreading of the manuscript and Daniel Dreier for processing of the medical images.

\section{References}

CVST as a Complication of a Microendoscopic Discectomy

\section{Disclosure Statement}

The authors declare that there is no conflict of interest that interferes with the publication of this report.

There are no financial conflicts of interest in this report.

Bousser MG, Ferro JM: Cerebral venous thrombosis: an update. Lancet Neurol 2007; $6: 162-170$

2 Martinelli I, Sacchi E, Landi G, Taioli E, Duca F, Mannucci PM: High risk of cerebral-vein thrombosis in carriers of a prothrombin-gene mutation and in users of oral contraceptives. N Engl J Med 1998;338: 1793-1797.

- 3 Filippidis A, Kapsalaki E, Patramani G, Fountas KN: Cerebral venous sinus thrombosis: review of the demographics, pathophysiology, current diagnosis, and treatment. Neurosurg Focus 2009;27:E3.
4 Mokri B: Spontaneous cerebrospinal fluid leaks: from intracranial hypotension to cerebrospinal fluid hypovolemia - evolution of a concept. Mayo Clin Proc 1999;74:1113-1123.

5 Bakshi R, Mechtler LL, Kamran S, et al: MRI findings in lumbar puncture headache syndrome: abnormal dural-meningeal and dural venous sinus enhancement. Clin Imaging 1999;23:73-76.

6 Ferro JM, Canhao P, Stam J, Bousser MG, Barinagarrementeria F: Prognosis of cerebral vein and dural sinus thrombosis: results of the International Study on Cerebral Vein and Dural Sinus Thrombosis (ISCVT). Stroke 2004;35:664-670. 